\title{
Retrospective Study Showing the Correlation between Morphine Use in Breast Cancer and the Incidence of Arrhythmia
}

\author{
Dharti Patel ${ }^{1, *}$, Urvi Patel ${ }^{2}$, Shikha Kothari ${ }^{3}$, Priya Rajpurohit ${ }^{4}$, Nirali Vassa ${ }^{1}$, Meghana Rao ${ }^{1}$, Ali Vaziri ${ }^{1}$, and Salman \\ Mudddassir ${ }^{1}$
}

${ }^{1}$ Oakhill Hospital Brooksville, United States

${ }^{2}$ Washington University St. Louis 63130, United States

${ }^{3}$ University of California Davis School of Medicine 95616, United States

${ }^{4}$ Pramukhswami Medical College Karamsad, India

*Corresponding author: Dharti Patel, Internal Medicine, Oakhill Hospital, 11375 Cortez Blvd Brooksville, FL 34613, United States, Phone: 269-769-4160; E-mail: dharti45@gmail.com

Received: 19 May, 2020 | Accepted: 01 Jul, 2020 | Published: 06 Jul, 2020

Citation: Patel D, Patel U, Kothari S, Rajpurohit P, Vassa N, et al. (2020) Retrospective Study Showing the Correlation between Morphine Use in Breast Cancer and the Incidence of Arrhythmia. J Breast Cancer Res Adv 2(1): dx.doi.org/10.16966/2638-3527.108

Copyright: (C) 2020 Patel D, et al. This is an open-access article distributed under the terms of the Creative Commons Attribution License, which permits unrestricted use, distribution, and reproduction in any medium, provided the original author and source are credited.

\section{Abstract}

Background: Patients with breast cancer may have an increase of a cardiac arrhythmia because of systemic inflammation induced by the cancer and side effects of treatments. The most common arrhythmia that has been linked with opiate use is atrial fibrillation. Atrial fibrillation has been known to have a high prevalence in the elderly population, and in correlation with congestive heart failure.

Objective: The purpose of this study was to establish the relationship between opiate use and incidence of having an arrhythmia in a patient with breast cancer compared with the background population.

Methods: This is a retrospective study using the HCA Enterprise Data Warehouse for the years 2016 to 2018. HCA is a private corporation that encompasses 185 hospitals and 119 freestanding surgery centers located in 21 states in the USA and the United Kingdom. The data was accumulated for the study using the International Classification of Diseases, $9^{\text {th }}$ Revision, Clinical Modification (ICD-9-CM) codes.

Results: We identified a malignancy cohort of 90,097 female breast cancer patients without any history of documented arrhythmia before the diagnosis of breast cancer between 2016-2018 by using the HCA data base in America. Compared with non-morphine users, patients who received any morphine exhibited a 2.117 -fold $(95 \% \mathrm{Cl}=1.864,2404)$ increase in developing an arrhythmia.

Conclusion: Our results indicate that there is an association of developing an arrhythmia with opiate use, in particular morphine. More studies are required to affirm this study before any conclusion can be drawn. Complications from arrhythmias should be monitored closed to prevent mortality.

Trial registration: This trial is not a prospective study. It is a retrospective study, retrospectively registered.

Keywords: Breast cancer; Opiates; Morphine; Arrhythmia; Atrial fibrillation

\section{Introduction}

Cancer patients are often administered morphine for pain alleviation; therefore, understanding the relationship between morphine use and arrhythmia incidence is important. We want to evaluate the potential for morphine-related arrhythmia, by comparing the incidence of arrhythmia in female patients with breast cancer treated with and without morphine. The most common arrhythmia that has been linked with opiate use is atrial fibrillation. Atrial fibrillation has been known to have a high prevalence in the elderly population, and also in correlation with congestive heart failure [1-3]. There has not been established data on the relevance to estrogen receptor and opiate use causing arrhythmia. There have been a very few number of trials associating opiate use inducing arrhythmias in female breast cancer patients. Our study contributes to the literature showing supporting evidence that opiate use, in particular morphine leads to an increase in an arrhythmia.

\section{Materials and Methods}

\section{Study population and design}

This is a retrospective study using the HCA Enterprise Data Warehouse for the years 2016-2018. HCA is a private corporation that encompasses 185 hospitals and 119 free-standing surgery centers located in 21 states in the USA and the United Kingdom. The data was accumulated for the study using the International Classification of Diseases, $9^{\text {th }}$ Revision, Clinical Modification (ICD9-CM) codes. 
Table 1: Demographics and relationship to opiate use.

\begin{tabular}{|c|c|c|c|c|c|}
\hline Variable & Arrhythmia=No & $\%$ & Arrhythmia=Yes & $\%$ & P Value \\
\hline Total & 90097 & & 1183 & & \\
\hline Age & & & & & $<0.0001$ \\
\hline $40-49$ & 12737 & 14.14 & 45 & 3.8 & \\
\hline $50-64$ & 36383 & 40.38 & 286 & 24.18 & \\
\hline $65-80$ & 40977 & 45.48 & 852 & 72.02 & \\
\hline Mean (SD) & 62.16 & 10.32(SD) & 68.68 & $8.58(S D)$ & $<0.0001$ \\
\hline Comorbidity & & & & & $<0.0001$ \\
\hline Hypertension & 14897 & 16.53 & 528 & 44.63 & $<0.0001$ \\
\hline Hyperlipidemia & 4928 & 5.47 & 334 & 28.23 & $<0.0001$ \\
\hline Hypothyroidism & 3141 & 3.49 & 176 & 14.88 & $<0.0001$ \\
\hline Diabetes type II & 6767 & 7.51 & 238 & 20.12 & $<0.0001$ \\
\hline Valvular heart disease & 2 & 0.00 & 1 & 0.08 & $<0.0001$ \\
\hline Heart failure & 935 & 1.04 & 120 & 10.14 & $<0.0001$ \\
\hline Estrogen positive & 10343 & 11.48 & 149 & 12.6 & 0.2322 \\
\hline Estrogen negative & 1758 & 1.95 & 24 & 2.03 & 0.8482 \\
\hline Anemia & 675 & 0.75 & 19 & 1.61 & 0.0007 \\
\hline Tobacco & 237 & 0.26 & 3 & 0.25 & 0.9497 \\
\hline Acute ischemic heart disease & 66 & 0.07 & 8 & 0.68 & $<0.0001$ \\
\hline COPD & 2304 & 2.56 & 120 & 10.14 & $<0.0001$ \\
\hline Alcohol abuse & 95 & 0.11 & 3 & 0.25 & 0.1221 \\
\hline Menopause & 9 & 0.01 & 0 & 0 & 0.7310 \\
\hline \multicolumn{6}{|l|}{ Opiates } \\
\hline Morphine & 16148 & 17.92 & 348 & 29.42 & $<0.0001$ \\
\hline Buprenorphine & 10 & 0.01 & 1 & 0.08 & 0.0223 \\
\hline Oxycdone & 2479 & 2.75 & 38 & 3.21 & 0.3364 \\
\hline Methadone & 101 & 0.11 & 2 & 0.17 & 0.5621 \\
\hline Tramdol & 0 & 0 & 0 & 0 & 0 \\
\hline
\end{tabular}

Table 2: Patient characteristics.

\begin{tabular}{|c|c|c|c|}
\hline Variable & $\begin{array}{c}\text { Atrial } \\
\text { fibrillation=No }\end{array}$ & $\begin{array}{c}\text { Atrial } \\
\text { fibrillation=Yes }\end{array}$ & P value \\
\hline \multicolumn{4}{|l|}{ Race } \\
\hline White & 63088 & 937 & $<0.0001$ \\
\hline Asian & 2071 & 13 & $<0.0001$ \\
\hline African-American & 11138 & 117 & $<0.0001$ \\
\hline Hispanic & 9309 & 88 & $<0.0001$ \\
\hline Other & 4491 & 28 & $<0.0001$ \\
\hline
\end{tabular}

\section{Inclusion criteria}

All adult (age $\geq 40$ years) patients hospitalized in 2016 without a known arrhythmia were identified. Using the ICD-9-CM code, individuals with breast cancer were identified and categorized. Female patients with breast cancer were subsequently identified (see Appendix for ICD-9-CM codes). Demographic characteristics were extracted, as well as comorbidities according to the Elixhauser comorbidity measure.

\section{Outcomes}

The primary outcome of our study was incidence of having arrhythmia in hospitalized patients with breast cancer with and without a concomitant use of opiates.

\section{Statistical analysis}

Descriptive statistics were used to summarize patient characteristics and comorbidities. Bivariate comparisons of patient characteristics, comorbidities, and outcomes between patients with breast cancer and opiate use were conducted using two-sided Chi Square and Independent $\mathrm{T}$ tests. Statistical significance was evaluated using $\alpha=0.05$. To further explore associations between arrhythmia and study outcomes, hyperthyroidism cases were propensity score-matched to controls based on sex, age, race, and comorbidity status. Statistical analyses were conducted using Statistical (version 13, Dell Inc.,) and R (version 3.6.1, The R Foundation for Statistical Computing) software.

\section{Results}

Our final dataset included 90,097 patients with breast cancer, 1183 of them had an arrhythmia, with the highest risk associated with morphine use. Table 1 further demonstrates the breakdown of breast cancer with type in patients with and without arrhythmias. However, no significant difference in the distribution of race between individuals with and without atrial fibrillation was observed. There was a difference in the opiates that were used. Morphine use showed a statistical significance with $\mathrm{p}<0.0001$ in regard to causing arrhythmia. There was no statistical significance in regards to a patient being estrogen receptor positive or negative. Patients who had any acute ischemic heart disease, heart failure, or even valvular disease showed statistical significance in regards with having an arrhythmia. These 
findings were consistent for propensity score matched atrial fibrillation cases and controls (Table 2).

\section{Discussion}

Breast cancer is one of the most common types of cancer and the third leading cause of cancer death in women [4]. From our knowledge, there have not been many studies correlating the use of opiates leading to an increase in arrhythmias in breast cancer. Cancer patients are often given morphine for relief of their pain and understanding any relationship between opiate uses is crucial. Approximately $30-50 \%$ of cancer patients have severe pain, and morphine remains the opiate of choice for pain alleviation [5].

Opioids are a drug class with pain relieving properties. They are well used in patients who have cancer. One of the most common side effects of opioids is their influence on the electrical activity of the heart the most common cardiac side effect of opioids is the prolongation of the QT interval, supra-ventricular arrhythmias, bradycardia, cardiac block and atrial fibrillation. Commonly used agents in cancer pain are methadone, tramadol, oxycodone, morphine, buprenorphine. Methadone is known to cause a greater risk of arrhythmias compared to tramadol and oxycodone which have intermediate risk. Morphine and buprenorphine are the two opiates that are low risk drugs known to cause arrhythmia [6].

One of the most common associated arrhythmias linked to breast cancer is atrial fibrillation. Atrial fibrillation is an arrhythmia that causes cardiac dysfunction by disorganized electrical signals causing unsynchronized contractility [7]. The reported associations between breast cancer and arrhythmia are not well understood. Advancing age is one risk factor. Obesity has been another reported factor. Cancer has been commonly associated with systemic inflammation. There is a recent hypothesis of genetics playing a role to explain the relationship between arrhythmia and cancer. Tumor suppressor genes protect cells from malignant transformation. Recent studies have shown that two tumor suppressor genes, Zinc finger homeobox protein-3 and esophageal cancer related gene- 4 are related to the development of atrial fibrillation [8]. Opium, a mixture of several opioids like morphine and codeine, is associated with AF as well, according to a few other studies. There has been one other famous study linking the relation with arrhythmia, specifically atrial fibrillation, which was done in Taiwan associated with morphine use [9]. This study showed that there was a correlation with incidence of arrhythmia, especially atrial fibrillation with opiate use.

\section{Limitations}

Our study has several limitations. The HCA database does not have a primary admission diagnosis. The patient could have been admitted for another complaint and not the diagnosis of breast cancer; the breast cancer could be a previous diagnosis. Secondly, we were not able to identify patients who were newly diagnosed with atrial fibrillation, or any other arrhythmia based on the electrocardiogram during the current admission as a part of their initial presentation with symptoms of breast cancer. Hence, these patients may not be a part of the data set.

\section{Conclusion}

We identified a malignancy cohort of 90,097 female breast cancer patients without any history of documented arrhythmia before the diagnosis of breast cancer between 2016-2018 by using the HCA data base in America. Compared with non morphine users, patients who received any morphine exhibited a 2.117 -fold $(95 \% \mathrm{CI}=1.864,2404)$ increase in developing an arrhythmia. Our results indicate that there is an association of developing an arrhythmia with opiate use, in particular morphine. More studies are required to affirm this study before any conclusion can be drawn. Complications from arrhythmias should be monitored closed to prevent mortality.

\section{Declarations}

\section{Ethical approval and consent to participate}

This research was supported (in whole or in part) by HCA Healthcare and/or an HCA Healthcare affiliated entity. The views expressed in this publication represent those of the author(s) and does not necessarily represent the official views of HCA Healthcare or any of its affiliated entities.

\section{Consent for Publication}

Not applicable.

\section{Availability of Data and Materials}

The datasets generated during and/or analysed during the current study are not publicly available due to being owned by HCA Company, but are available from the corresponding author on reasonable request.

\section{Competing Interests}

The authors declare that they have no competing interests in this section.

\section{Funding}

There was no funding for this research.

\section{Authors' Contributions}

Dharti Patel, Nirali Vassa, Meghana Rao, Salman Muddassir-data collection, analysis, writing manuscript.

Urvi Patel, Shikha Kothari, Priya Rajpurohit-editing.

\section{Acknowledgements}

Not applicable.

\section{Authors Information}

Not applicable.

\section{References}

1. Schnabel RB, Yin X, Gona P, Larson MG, Beiser AS, et al. (2015) 50 Year Trends in Atrial Fibrillation Prevalence, Incidence, Risk Factors, and Mortality in the Framingham Heart Study: A Cohort Study. Lancet 386: 154-162.

2. Wang CC, Lin CL, Wang GJ, Chang CT, Sung FC, et al. (2015) Atrial Fibrillation Associated with Increased Risk of Venous Thromboembolism. A Population-Based Cohort Study. Thromb Haemost 113: 185-192.

3. Hui DS, Morley JE, Mikolajczak PC, Lee R (2015) Atrial Fibrillation: A Major Risk Factor for Cognitive Decline. Am Heart J 169: 448-456.

4. Siegel RL, Miller KD, Jemal A (2020) Cancer statistics, 2020. CA Cancer J Clin 7: 1-30.

5. Wiffen PJ, Wee B, Moore RA (2013) Oral Morphine for Cancer Pain. Cochrane Database Sys Rev 7: CD003868.

6. Behzadi M, Joukar S, Beik A (2018) Opioids and Cardiac Arrhythmia: A Literature Review. Med Princ Pract 27: 401-414.

7. Jalife J, Kaur K (2015) Atrial Remodeling, Fibrosis, and Atrial Fibrillation. Trends Cardiovasc Med 25: 475-484. 
8. Mao L, Huang W, Zou P, Dang X, Zeng X (2018) The Unrecognized Role of Tumor Suppressor Genes in Atrial Fibrillation. Gene 642: 2631.

9. Lee CW, Muo CH, Liang JA, Lin MC, Kao CH (2016) Atrial Fibrillation is Associated With Morphine Treatment in Female Breast Cancer Patients: A Retrospective Population-Based Time-Dependent Cohort Study. Medicine (Baltimore) 95: e3102. 